\title{
Panobinostat Nanoparticle Formulation MTX110
}

National Cancer Institute

\section{Source}

National Cancer Institute. Panobinostat Nanoparticle Formulation MTX110. NCI

Thesaurus. Code C150717.

A gold nanoparticle (GNP)-based formulation containing panobinostat, a pan histone deacetylase (HDAC) inhibitor, with potential antineoplastic activity. Upon intra-tumoral injection of MTX110, panobinostat is released from the formulation and selectively targets, binds to and inhibits histone deacetylase (HDAC), which induces hyperacetylation of core histone proteins. The accumulation of highly acetylated histones leads to chromatin remodeling, an altered pattern of gene expression, inhibition of tumor oncogene transcription and the selective transcription of tumor suppressor genes. This results in the inhibition of tumor cell division and the induction of tumor cell apoptosis. HDAC, upregulated in many tumor cell types, is an enzyme family that deacetylates histone proteins. Panobinostat is water insoluble and does not cross the blood-brain barrier (BBB) when administered orally or intravenously. MTX110 solubilizes panobinostat and can be directly injected into the brain, which bypasses the BBB and delivers high concentrations of panobinostat to the tumor, while minimizing systemic toxicity. 